\title{
Continuous Cerebral and Myocardial Selective Perfusion in Neonatal Aortic Arch Surgery
}

\author{
Pierre Maminirina ${ }^{1}$, Carine Pavy $^{1}$, Pierre Bourgoin ${ }^{1}$, and Olivier Baron ${ }^{1}$ \\ ${ }^{1}$ University Hospital Centre Nantes
}

May 18, 2020

\begin{abstract}
BACKGROUND: To assess the feasibility and outcome of Continuous Cerebral and Myocardial Selective Perfusion (CCMSP) during aortic arch surgery in neonates. METHODS: This retrospective single-center study was conducted between 2008 and 2019 in neonates undergoing aortic arch surgery, accompanied or not by cardiac malformation repair. CCMSP at moderate hypothermic of $28^{\circ} \mathrm{C}$ was achieved using selective brachiocephalic artery and ascending aorta cannulation. Target rates of cerebral and myocardial perfusion were $25-35 \mathrm{~mL} / \mathrm{kg} / \mathrm{min}$ and $150 / \mathrm{m} 2 / \mathrm{min}$. Cardiopulmonary bypass (CPB) variables and clinical outcomes were analyzed. RESULTS: Overall, 69 neonates underwent either isolated aortic arch repair $(\mathrm{n}=31)$ or aortic arch repair with ventricular septal defect (VSD) closure $(\mathrm{n}=38)$. The mean age and weight were $9.79 \pm 7.1$ days and $3.17 \pm 0.4 \mathrm{Kg}$, respectively. Mean CPB and aortic clamping times were $133.5 \pm 47.0$ and $25.2 \pm 5.3 \mathrm{~min}$ for isolated aortic arch repair, and $158.4 \pm 47.9$ and $75.4 \pm 30.5 \mathrm{~min}$ for aortoplasty accompanied by VSD closure. Mean CCMSP time was $51.6 \pm 21.5$ min with cerebral rate of $32.6 \pm 10.0 \mathrm{~mL} / \mathrm{Kg} / \mathrm{min}$. Two major complications arose: stroke $(\mathrm{n}=1 ; 1.44 \%)$ and transient renal failure requiring dialysis $(\mathrm{n}=2$; $2.89 \%)$. Neither myocardial nor visceral ischemia occurred. In-hospital mortality was 1/69 (1.44\%). CONCLUSIONS: CCMSP is a safe and reproducible strategy for cerebral, myocardial and visceral protection in neonatal aortic arch repair, with or without VSD closure, resulting in low complication and mortality.
\end{abstract}

\section{INTRODUCTION}

Aortic arch surgery presents a challenge in neonates in terms of organ protection. Deep hypothermic circulatory arrest (DHCA) has so far been the most common adjunctive support technique. While this technique protects vital organs due to hypothermia, it has the added technical advantage of removing blood from the operative field (1). Though DHCA provides time-limited protection, it is not devoid of damaging effects (2). Though still a mainstay in congenital heart surgery, DHCA is associated with poor neurodevelopmental outcome (3). Alternative perfusion techniques have, therefore, been developed, such as antegrade cerebral perfusion (ACP) combined with moderate hypothermic circulatory arrest (MHCA) of the lower part of the body (4). This study sought to evaluate the feasibility and efficacy of combined continuous cerebral and myocardial selective perfusion strategy (CCMSP) as applied in our center.

\section{METHODS}

\section{Patient population}

Between 2008 and 2019, 69 consecutive neonates underwent aortic arch reconstruction, with or without intracardiac repair, in our center. All presented with: coarctation of the aorta (CoA) with ventricular septal defect (VSD), severe hypoplastic aortic arch (HAA) with or without VSD, interrupted aortic arch (IAA). Their medical charts and surgical reports were retrospectively analyzed. All neonates were undergoing their first operation. All complex congenital malformation (Hypoplastic left heart syndrome, Common arterial trunk...) and surgical re-interventions were excluded from analysis. Table 1 summarizes population characteristics and lesion distribution. 


\section{Surgical technique}

Following median sternotomy and thymus removal, an autologous pericardial patch was harvested and fixed in glutaraldehyde. Figure 1 summarizes surgical technique applied in our center. The brachiocephalic artery (BCA) was then cannulated using a Gore-Tex tube, and accomplished bicaval venous cannulation. On initiating cardiopulmonary bypass (CPB), core cooling was commenced. We tightened the clamps on the pulmonary arteries to maximize body perfusion via the ductus arteriosus, while continuing the preparatory dissection as necessary. When the rectal temperature reached $28^{\circ} \mathrm{C}$, circulatory arrest in the lower part of the body was begun by clamping the BCA at its origin. The CPB flow rate was lowered to $25-35 \mathrm{~mL} / \mathrm{Kg} / \mathrm{min}$ to ensure continuous ACP via the BCA. We clamped the left subclavian artery and left common carotid artery to optimize intracerebral perfusion pressure. At that point, our target was a right radial artery blood pressure of $>50 \mathrm{mmHg}$, and a bilateral near-infrared spectroscopy value of $>50 \%$. Anesthesia was induced using an intravenous midazolam injection, with electrolyte and acid-base balances managed using the alphastat approach. We first clamped the descending aorta followed by the ascending aorta. At that point, we perfused the coronary arteries with blood at $32^{\circ} \mathrm{C}$ with a rate of $150 \mathrm{ml} / \mathrm{m} 2 / \mathrm{min}$. We divided the ascending aorta just prior to the BCA's origin, then ligated and divided the ductus arteriosus, resecting as much ductal tissue as possible. The hypoplastic aortic arch was longitudinally opened, and all four ends exposed. Next, we enlarged the arch using the autologous pericardial patch as an aortic tube according to z-score normal size. Then, we performed end-to-end anastomosis on the descending aorta to restore the continuity of the arch and descending aorta. The heart had thus far been kept beating during the procedure. At this point, we induced cardioplegia using a potassium-enriched solution to arrest the heart. Next, we removed the clamp from the ascending aorta, re-established the continuity of the ascending aorta to aortic arch and respecting its good concavity shape. We then provoked retrograde purging of the aorta and we gradually increased the $\mathrm{CPB}$ flow rate, removing the clamp from the $\mathrm{BCA}$. The $\mathrm{CPB}$ was then resumed, with the flow rate calculated to ensure systemic patient perfusion. Before proceeding to rewarming, we carefully checked for any hemorrhaging. Next, we performed a possible additional repair during the rewarming period, such as VSD closure. We weaned the $\mathrm{CPB}$ when the rectal temperature reached $36.5^{\circ} \mathrm{C}$. We administered hemostatic products to ensure good hemostasis. The sternum was initially left open if necessary. Nowadays, sternum is usually closed immediately

\section{RESULTS}

\section{Duration of CPB and aortic clamping}

Table 2 illustrates the duration of $\mathrm{CPB}$ and aortic clamping according to surgical repair procedure. The mean durations of CPB and aortic clamping upon aortoplasty were 133.5 \pm 47.0 and $25.2 \pm 5.3$ minutes. Longer durations were observed in case of VSD closure. For these, the mean durations of CPB and aortic clamping were $158.4 \pm 47.9$ and $75.4 \pm 30.5$ minutes respectively.

\section{Cerebral and myocardial perfusion}

Table 3 provides the parameters recorded during CCMSP. The mean duration of CCMSP was $51.6 \pm 21.5$ minutes (median: 52). The mean cerebral perfusion flow rate was $32.6 \pm 10.0 \mathrm{~mL} / \mathrm{Kg} / \mathrm{min}$. The mean cooling time was $22.5 \pm 1$ minutes with a median of 21 minutes whereas the mean rewarming time was $42.6 \pm 16.3$ minutes with a median of 40 minutes. Arterial perfusion and venous return temperatures were practically identical: 26.4 vs. $26.9^{\circ} \mathrm{C}$. The rate of myocardial perfusion was constant at $150 \mathrm{ml} / \mathrm{m} 2 / \mathrm{min}$ according to our protocol.

\section{Major complications}

\section{Neurological complications}

One case of stroke $(1.44 \%$ ) occurred in a male neonate aged 30 days and weighing $3 \mathrm{Kg}$, with severe HAA and VSD, admitted to our pediatric intense care unit in a state of cardiogenic shock. His hemodynamics improved upon inotropic medication, and surgery was performed 24 hours post-admission, consisting of complete reconstructive aortoplasty and VSD closure. CPB lasted 181 minutes, aortic clamping 90 minutes, 
and CCMSP of 55 minutes. After weaning from CPB failed, the neonate was placed on central extracorporeal membrane oxygenation (ECMO), and then withdrawn from ECMO after 7 days. Thereafter, the infant displayed marked global developmental delay, brain imaging revealing ischemic and hemorrhagic lesions in the frontal and occipital regions.

\section{Renal complications}

Two (2.89\%) neonates developed transient renal failure requiring dialysis with patients' characteristics listed in Table 4. They weighed less than $3 \mathrm{~kg}$ underwent total reconstruction for HAA and concomitant VSD with CPB for over 150 minutes. Durations of CCMSP were 42 and 46 minutes.

No myocardial or visceral ischemia complications were observed in our study.

\section{In-hospital mortality}

The mortality rates were 1.44\% (1/69). The cause of death was a septic shock Escherichia coli-related.

\section{DISCUSSION}

\section{Surgical treatment indication and approach}

All surgical practitioners in our center followed the same protocol for aortic arch surgery. We have a firm preference for performing one-step complete arch reconstruction in neonates with CoA with VSD. Within the Society of Thoracic Surgeons (STS) Congenital Heart Surgery database, Ross and colleagues (5) identified 840 patients treated for CoA with VSD, 211 (25\%) of whom underwent VSD closure and 89 (11\%) pulmonary artery banding, whereas 540 (64\%) did not undergo VSD closure. In this study, in-hospital mortality proved low, without significant between-strategy differences. In our center, HAA or CoA without VSD are treated differently, depending on the lesion extent. Arch enlargement plasty via median sternotomy is our preferred approach for severe proximal HAA cases. This also applies to any aortic Segment I hypoplasia with a Zscore $<-3$ and diameter inferior to body weight $(\mathrm{Kg})+1 \mathrm{~mm}$. Similarly, we prefer this approach for managing supra-aortic artery abnormalities like bovine arch. Complete reconstruction via median sternotomy and CPB enables good arch reconstruction without requiring aortic clamping at the BCA's origin. Of note, median sternotomy enables us to simultaneously close any associated atrial septal defect, thereby improving left ventricular preload. Optimizing this preload further improves left ventricular development, and that of the aorta downstream, potentially reducing reoperation rates (6). The Sakurai and colleagues' study involved 288 patients treated for CoA and HAA without VSD over two decades. Their analysis revealed a trend towards a higher number of sternotomies during the second decade (36\% vs. $6 \%$ ), along with a significantly low reoperation rate $(5 \%$ vs. $16 \%, \mathrm{p}=0.02)$ (7). Complete reconstruction via lateral thoracotomy, using the extended Crafoord technique (8) without CPB, is the approach we prefer in case of isolated isthmic CoA, either small in size or extending as far as Segment II .Over a 10-year period (2008-2019), we performed 180 lateral thoracotomies versus 31 median sternotomies, all age groups taken together.

\section{Surgical procedure and perfusion technique}

Generally, we select the perfusion technique depending on the patient's age and aortic arch repair complexity. CCMSP at $28^{\circ} \mathrm{C}$ is our preferred technique for all extensive aortic arch reconstruction in neonates. DHCA is rarely performed, rather reserved for more complex repairs. Whole-body perfusion at normothermia without circulatory arrest, indicated for older children, is achieved by perfusing the brain via the BCA and the body via femoral artery cannulation. According to the literature, the perfusion techniques in pediatric aortic arch surgery vary widely and the debate is never ending, with no established consensus as of yet. MHCA with ACP is likely to provide improved brain protection with fewer neurological complications (2)(3)(9) though several authors were unable to clearly demonstrate ACP's superiority over DHCA in terms of neurocognitive complications. In a randomized controlled trial comparing DHCA versus ACP, Algra and colleagues did not detect any between-group difference in new neurological injuries on immediate post-operative MRI or in neurocognitive or motor outcomes at 24 months (10). Using the STS database with 4,523 American patients, Meyer and colleagues reported ACP to have increasingly become the new trend, used $43 \%$ of times versus 
$32 \%$ for DHCA (11). Approximately $16 \%$ of centers employ a mixed approach comprising both a complete circulatory arrest and an ACP during the same procedure.

\section{Target temperature and flow rate}

In our cohort, we opted for a rectal target temperature of $28^{\circ} \mathrm{C}$. This target temperature was perfectly aligned with the CPB temperatures recorded during circulatory arrest of the lower part of the body, attesting to a relatively stable hypothermia. Mean venous return and arterial perfusion temperatures were $26.9^{\circ} \mathrm{C}$ and $26.4^{\circ} \mathrm{C}$, with medians of $27^{\circ} \mathrm{C}$ and $26.5^{\circ} \mathrm{C}$ during CCMSP. According to our calculations, an ACP flow rate of $25-35 \mathrm{~mL} / \mathrm{Kg} / \mathrm{min}$ was adequate, whereas the mean flow rate recorded was $32.6 \pm 10.0 \mathrm{~mL} / \mathrm{kg} / \mathrm{min}$, with a median of $30.5 \mathrm{~mL} / \mathrm{Kg} / \mathrm{min}$. A temperature of $28^{\circ} \mathrm{C}$ and ACP flow rate of $25-35 \mathrm{~mL} / \mathrm{Kg} / \mathrm{min}$ had been fixed as objectives, based on our center's experience in adult aortic surgery and several studies involving hypothermic pump flow rates and blood gas management published in the literature (12)(13)(14). Our target parameters for cerebral and visceral protection are: right radial artery blood pressure $>50 \mathrm{mmHg}$, bilateral near infrared spectroscopy (NIRS) values $>50 \%$, transcranial Doppler flow velocity $>15-20 \mathrm{~cm} / \mathrm{s}$, and mixed venous oxygen saturation $>60 \%$. Body temperatures and cerebral perfusion rates during circulatory arrest are a matter of endless debate. Several authors have performed ACP during normothermia, aiming to achieve better protection by staying as close as possible to the normal human physiological state. To maintain normothermia, they are now experimenting with splanchnic perfusion to prevent renal and gut exposure to circulatory arrest. These authors have reported satisfactory results in terms of visceral and neurological function (15). Notably, Fraser and colleague recommended a higher ACP flow rate at $50-80 \mathrm{~mL} / \mathrm{Kg} / \mathrm{min}$ (14). Nevertheless, our strategy of applying CCMSP at $28^{\circ} \mathrm{C}$ with flow rate of $25-35 \mathrm{~mL} / \mathrm{Kg} / \mathrm{min}$ has so far yielded suitable results, which will be discussed in more detail later.

\section{Myocardial protection}

In all cases, we performed continuous blood coronary perfusion, in an effort to maintain the heart beating during aortic arch repair. To maximize myocardial protection, we shortened the duration of cardiac arrest as much as possible. We reserve cardiac arrest to perform our aorta proximal anastomosis in order to achieve a good, extensive, arch-shaped augmentation. The duration of cardiac arrest can be extended for any additional repair procedure. In their study, Lim and colleagues compared two perfusion groups involving 97 neonates operated on for CoA and HAA with VSD, IAA with VSD or aortic abnormality associated with complex cardiac malformations (16). In all cases, simultaneous cerebral and myocardial perfusion displayed superiority in terms of vasopressin use and extubation versus cerebral perfusion alone. In the most complex cases, simultaneous cerebral and myocardial perfusion was additionally shown to be superior in terms of vasoactive inotrope use, secondary sternal closure, removal of chest tubes, pleural effusion, and ICU and hospital stay duration. In our center, we apply a patch of autologous pericardium as augmentation material, preferring this approach to the direct resection/anastomosis technique. This patch technique enables us to achieve a good post-repair arch shape. While this procedure may slightly prolong the reparation in comparison with direct resection/anastomosis, we favor the anatomical outcome, along with good visceral and cerebral protection, over simple circulatory arrest duration. Our mean CCMSP duration of 51.6 \pm 21.5 minutes is roughly in line with that of the literature. In the aforementioned Meyer report using the STS database, the mean circulatory arrest duration with cerebral perfusion was 45.0 (30.0-63.0) minutes (12).

\section{Major complications and mortality}

Our study generated very promising results concerning mortality (1.44\%) and major complications, with only one case $(1.44 \%)$ of neurological injury and two cases $(2.89 \%)$ of transient renal failure requiring dialysis. No myocardial or visceral ischemic injuries were observed. Currently, aortic arch repair with or without VSD repair using median sternotomy and CPB is a well-mastered technique. Despite long CPB or circulatory arrest times in several cases, CCMSP at moderate hypothermic circulation was able to ensure good organ protection. Despite of the circulatory arrest of the lower part of the body, organ perfusion are ensured by multiple vascular communication through the selective cerebral perfusion. That's why we clamp the descending aorta during the aortic arch reparation to avoid back flow. On note is that we do not perform systematic brain imaging after 
arch surgery, reserving imaging for children with clinical neurological disorders. Overall, our clinical results are very satisfactory. Concerning cerebral protection, Rüffer and colleagues demonstrated symmetrical perfusion of both cerebral hemispheres during ACP (17), while Algra and colleagues clearly illustrated how continuous cerebral perfusion during neonatal aortic arch surgery caused decreased circulatory arrest-induced renal and visceral injury (18). Comparing the two perfusion techniques performed at $18^{\circ} \mathrm{C}$, the latter study reported that ACP was superior to DHCA in terms of abdominal perfusion, as confirmed by near-infrared spectroscopy data, postoperative inferior vena cava lactate levels, and renal and gastrointestinal injury biomarkers.

\section{Limitations and strengths}

This study's limitations lay in its retrospective nature and patient population's heterogeneity, with study data solely derived from patients' hospital charts. We also lacked laboratory data or imaging reports enabling us to identify subclinical abnormalities. Moreover, the neurological assessment was solely based on clinical findings followed-up by imaging, without any established imaging protocol applied. The next necessary step is now a prospective study using exhaustive data collection according to a pre-established protocol to fully assess this surgery and perfusion technique.

\section{CONCLUSION}

Aortic arch surgery during the neonatal period still poses a challenge in terms of feasibility and outcome. Choosing the appropriate perfusion technique is vital for ensuring better long-term outcomes. There is no consensus yet as to either the perfusion type or the temperature and flow rates of cerebral perfusion. Dual selective cerebral and coronary continuous perfusion at moderate hypothermic circulatory appears feasible, reliable, and reproducible, while ensuring maximal cerebral and visceral protection, without exposure to ischemic complications.

\section{REFERENCES}

1. Castaneda AR, Mayer JE Jr, Jonas RA, Lock JE, Wessel DL, Hickey PR. The neonate with critical congenital heart disease: repair-a surgical challenge. J Thorac Cardiovasc Surg. 1989;98:869-875.

2. Newburger JW, Jonas RA, Wernovsky G, Wypij D, Hickey PR, Kuban KC et al. A comparison of the perioperative neurologic effects of hypothermic circulatory arrest versus low-flow cardiopulmonary bypass in infant heart surgery. N Engl J Med. 1993;329:1057-1064.

3. Oates RK, Simpson JM, Turnbull JAB, Cartmill TB. The relationship between intelligence and duration of circulatory arrest with deep hypothermia. J Thorac Cardiovasc Surg. 1995;110:786-792.

4. Pigula FA, Siewers RD, Nemoto EM. Regional perfusion of the brain during neonatal aortic arch reconstruction. J Thorac Cardiovasc Surg 1999;117:1023-1024.

5. Ross M. Ungerleider, Sara K. Pasquali, Karl F. Welke et al. Contemporary patterns of surgery and outcomes for aortic coarctation: An analysis of the Society of Thoracic Surgeons Congenital Heart Surgery Database. J Thorac Cardiovasc Surg. 2013; 145: 150-157.

6. Rüffer A, Bechtold C, Purbojo A, Toka O, Glöckler M, Dittrich etal. Aortic arch obstruction neonates with biventricular physiology: left-open compared to closed inter-atrial communication during primary repair-a retrospective study. J Cardiothorac Surg. 2015;17;10:53.

7. Sakurai T, Stickley J, Stümper O, Khan N, Jones TJ, Barron DJ et al. Repair of isolated aortic coarctation over two decades: impact of surgical approach and associated arch hypoplasia. Interact Cardiovasc Thorac Surg. 2012;15: 865-870.

8. Crafoord C, Nylin G. Congenital coarctation of the aorta and its surgical treatment. J Thorac Surg. 1945; 14: $347-361$.

9. Andropoulos DB, Easley RB, Brady K, McKenzie ED, Heinle JS, Dickerson HA, et al. Neurodevelopmental outcomes after regional cerebral perfusion with neuromonitoring for neonatal aortic arch reconstruction. Ann 
Thorac Surg. 2013;95: 648-654.

10. Algra SO, Jansen NJ, van der Tweel I, Schouten AN, Groenendaal F, Toet M et al. Neurological injury after neonatal cardiac surgery: a randomized, controlled trial of 2 perfusion techniques. Circulation 2014;129:224-33.

11. Meyer DB, Jacobs JP, Hill K, Wallace AS, Bateson B, Jacobs ML. Variation in Perfusion Strategies for Neonatal and Infant Aortic Arch Repair: Contemporary Practice in the STS Congenital Heart Surgery Database.World J Pediatr Congenit Heart Surg. 2016;7:638-644.

12. Sasaki T, Tsuda S, Riemer RK, Ramamoorthy C, Reddy VM, Hanley FL. Optimal flow rate for antegrade cerebral perfusion. J Thorac Cardiovasc Surg 2010; 139: 530-535.

13. Oppido G, Pace Napoleone C, Turci S, Davies B, Frascaroli G, Martin-Suarez S et al. Moderately hypothermic cardiopulmonary bypass and low-flow antegrade selective cerebral perfusion for neonatal aortic arch surgery. Ann Thorac Surg. 2006; 82 :2233-2239.

14. Fraser CD Jr, Andropoulos DB. Principles of antegrade cerebral perfusion during arch reconstruction in newborns/infants. Semin Thorac Cardiovasc Surg Pediatr Card Surg Annu. 2008:61-68.

15. Raees MA, Morgan CD, Pinto VL, Westrick AC, Shannon CN, Christian KG et al. Neonatal Aortic Arch Reconstruction With Direct Splanchnic Perfusion Avoids Deep Hypothermia. Ann Thorac Surg. 2017;104:2054-2063

16. Lim HG, Kim WH, Park CS, Chung ES, Lee CH, Lee JR et al. Usefulness of Regional Cerebral Perfusion Combined With Coronary Perfusion During One-Stage Total Repair of Aortic Arch Anomaly. Ann Thorac Surg. 2010;90:50 -57.

17. Rüffer A, Tischer P, Münch F, Purbojo A, Toka O, Rascher W et al. Comparable Cerebral Blood Flow in Both Hemispheres During Regional Cerebral Perfusion in Infant Aortic Arch Surgery. Ann Thorac Surg. 2017;103:178-185.

18. Algra SO, Schouten AN, van Oeveren W, van der Tweel I, Schoof PH, Jansen N et al. Low-flow antegrade cerebral perfusion attenuates early renal and intestinal injury during neonatalaortic arch reconstruction. J Thorac Cardiovasc Surg. 2012;144:1323-8, 1328

\section{Figure and Table legends}

Table 1: Patient demographics and diagnosis. VSD: Ventricular septal defect; SD: standard deviation

Figure 1 : Surgical technique.1)BCA cannulation. 2) Circulatory arrest at $28^{\circ} \mathrm{C}$; antegrade cerebral perfusion: $25-35 \mathrm{~mL} / \mathrm{Kg} / \mathrm{min}$; clamping of BCA, left common carotid artery, left subclavian artery. 3) Clamping of ascending aorta and descending aorta. 4) Aortoplasty using autologous pericardial patch. 5) Blood coronary perfusion without cardioplegia. 6) Proximal anastomosis; open aorta; cardiac arrest. 7) Systemic flow rate reestablished. 8) VSD closure. Note: The red arrows indicate the blood flow direction. BCA:brachiocephalic artery; VSD: ventricular septal defect.

Table 2 : CPB and aortic clamping according to repair procedure.CPB: Cardiopulmonary bypass, VSD: Ventricular septal defect

Table 3 : Parameters recorded during CCMSP

Table 4 : Characteristics of neonates with kidney complications requiring dialysis. HAA: Hypoplastic aortic arch, VSD: Ventricular septal defect, CPB: Cardiopulmonary bypass

\section{Hosted file}

Tables 1.doc available at https://authorea.com/users/322641/articles/451592-continuouscerebral-and-myocardial-selective-perfusion-in-neonatal-aortic-arch-surgery 


\section{Hosted file}

Figure 1.docx available at https://authorea.com/users/322641/articles/451592-continuouscerebral-and-myocardial-selective-perfusion-in-neonatal-aortic-arch-surgery

\section{Hosted file}

Table 2.docx available at https://authorea.com/users/322641/articles/451592-continuouscerebral-and-myocardial-selective-perfusion-in-neonatal-aortic-arch-surgery

\section{Hosted file}

Table 3.docx available at https://authorea.com/users/322641/articles/451592-continuouscerebral-and-myocardial-selective-perfusion-in-neonatal-aortic-arch-surgery

\section{Hosted file}

Table 4.docx available at https://authorea.com/users/322641/articles/451592-continuouscerebral-and-myocardial-selective-perfusion-in-neonatal-aortic-arch-surgery 